Provided for non-commercial research and education use. Not for reproduction, distribution or commercial use.

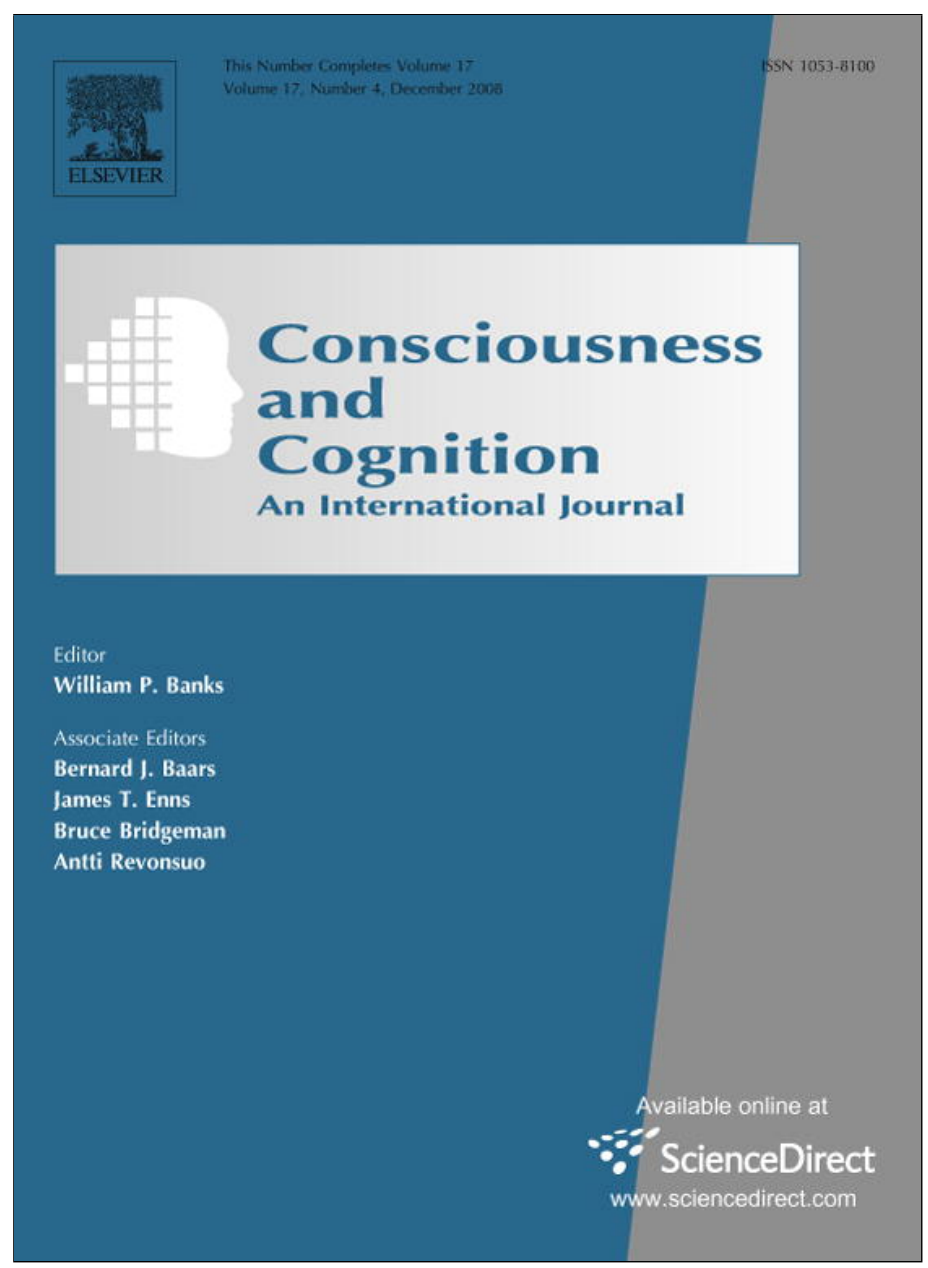

This article appeared in a journal published by Elsevier. The attached copy is furnished to the author for internal non-commercial research and education use, including for instruction at the authors institution and sharing with colleagues.

Other uses, including reproduction and distribution, or selling or licensing copies, or posting to personal, institutional or third party websites are prohibited.

In most cases authors are permitted to post their version of the article (e.g. in Word or Tex form) to their personal website or institutional repository. Authors requiring further information regarding Elsevier's archiving and manuscript policies are encouraged to visit:

http://www.elsevier.com/copyright 
Consciousness

and

Cognition

www.elsevier.com/locate/concog

\title{
Conscious and unconscious proportion effects in masked priming is
}

\author{
Eva Van den Bussche *, Gitte Segers, Bert Reynvoet \\ Department of Psychology and Educational Sciences, University of Leuven Campus Kortrijk, E. Sabbelaan 53, 8500 Kortrijk, Belgium
}

Received 5 April 2007

Available online 4 October 2007

\begin{abstract}
The global neuronal workspace theory [Dehaene, S., \& Naccache, L. (2001). Towards a cognitive neuroscience of consciousness: basic evidence and a workspace framework. Cognition, 79, 1-37.] proposes that it is impossible for an unconscious stimulus to be used strategically to enhance task performance (bottom-up effect), while a fully consciously perceived stimulus can be used to improve task performance (top-down effect). Two experiments were designed to investigate these hypotheses. In a first experiment we investigated whether a manipulation of the proportion of Arabic/number word targets had a top-down effect. Since the subjects were fully aware of the target, we expected that this manipulation could be used to enhance task performance. In a second experiment we determined whether a manipulation of the proportion of Arabic/ number word primes had a bottom-up effect. Since the subjects were unaware of the prime, we hypothesized that they would be unable to use the manipulation to enhance task performance and therefore the manipulation would have no effect. Both hypotheses were largely confirmed.
\end{abstract}

(c) 2007 Elsevier Inc. All rights reserved.

Keywords: Masked priming; Proportion effect; Bottom-up effect; Top-down effect

\section{Introduction}

Many theories of human cognition postulate that unconscious cognitive processes, such as unconscious priming, are automatic and independent of attention. According to these traditional accounts, unconscious processing is confined to a passive spreading of activation, inflexible and independent of the subject's attention and strategies. Recently, Dehaene and colleagues (Dehaene, Changeux, Naccache, Sackur, \& Sergent, 2006) defined subliminal processing as "a condition of information inaccessibility where bottom-up activation is insufficient... for global ignition" (p. 3). However, they stress that even though the bottom-up strength of

\footnotetext{
Eva Van den Bussche and Bert Reynvoet are affiliated to the Laboratory of Experimental Psychology of the Department of Psychology, University of Leuven and to the Subfaculty of Psychology and Educational Sciences, University of Leuven-Campus Kortrijk. We thank three anonymous reviewers for their helpful comments and suggestions.

* Corresponding author. Fax: $+32(0) 56246052$.

E-mail address: Eva.Vandenbussche@kuleuven-kortrijk.be (E. Van den Bussche).
} 
subliminal stimuli is limited, top-down manipulations can have an effect on subliminal processing, indicating that subliminal processing is not merely a matter of automatic activation spreading. Indeed, a number of experimental reports using the masked priming paradigm have revealed that unconscious processing is affected by several top-down effects. Unconscious priming has been shown to be influenced by temporal attention (e.g., Naccache, Blandin, \& Dehaene, 2002), spatial attention (e.g., Sumner, Tsai, Yu, \& Nachev, 2006) and strategies, such as target set (e.g., Greenwald, Abrams, Naccache, \& Dehaene, 2003) and target notation (Kunde, Kiesel, \& Hoffmann, 2003). For instance, this latter top-down effect of target notation was investigated by Kunde and colleagues (2003, experiment 4). They showed that priming effects elicited by unconscious Arabic number primes (i.e. 1, 4,..) and number word primes (i.e. One, Four,...) were dependent on the target notation. If all targets were Arabic numbers, only Arabic number primes elicited a congruency effect. Similarly, if the targets were number words, only number word primes evoked an effect. According to the authors, subjects create intentions based on the stimulus characteristics and apply these intentions to the processing of the primes, which is clearly a top-down influence.

Although several studies provide strong evidence in favour of top-down influencing of unconscious priming by several factors, the conducted research is less clear on whether unconscious primes can themselves be used strategically and result in a bottom-up effect. Theoretically, the possibility of such a bottom-up effect of unconscious stimuli has been refuted. The definition of Dehaene et al. (2006), stating that unconscious primes have insufficient strength to result in a bottom-up effect, is situated within a broader framework proposed by Dehaene and Naccache (2001). They attempted to formulate a theoretical framework on consciousness based on a cognitive neuroscience approach. Their global neuronal workspace theory distinguishes two computational spaces in the brain (Dehaene \& Changeux, 2004): (1) the network of processors, which is a collection of specialized processors all dedicated to the processing of very specific information and (2) the global neuronal workspace (GNW) which, contrary to the local and modular processors, allows many different processors to exchange information in a global and flexible way. The GNW is assumed to play a crucial role in accessing information into consciousness. A mechanism called topdown attentional amplification, situated in the GNW, can activate local processors in the global workspace. This top-down process is necessary for information to access consciousness. However, to enter consciousness, a processor must not only be activated in the GNW, this activity must also be amplified and maintained over a minimal duration to reach the consciousness threshold. This implies that there are two thresholds in human information processing: (1) the minimal duration to reach any neural activity at all, and (2) the significantly longer duration to reach the consciousness threshold. Stimuli that fall in between these two thresholds can cause unconscious processing, but can never reach a conscious state. A longer or stronger stimulation is required to reliably activate the GNW, necessary to reach consciousness. Contrarily, a masked stimulus, which reaches the first threshold, but not the second one, can result a burst of activity, but only a weak and short-lived activation of the GNW is seen. In a realistic situation however, unconscious processing does not have to be as rigid as described above and context effects come into play. Based on this theoretical framework, the GNW model makes two basic assumptions about the possibility of conscious and unconscious (context) stimuli to result in a top-down and/or a bottom-up effect. First, explicit context stimuli (e.g., task instructions or target manipulations as described at the beginning of the introduction) can induce a reorganization of the GNW through top-down amplification. The GNW theory predicts that such reorganization can effect the processing of unconscious information, as long as the context stimuli which guide this process themselves are conscious. Thus, when a task is prepared consciously, it can be applied unconsciously. This first theoretical assumption has been confirmed by a number of research results, as we described above: several explicit top-down manipulations have proven to influence unconscious processing.

Second, if initially unattended stimuli carry enough strength, they can cause a reorienting of top-down amplification towards them, which is called a bottom-up effect. However, since for such a bottom-up effect to take place conscious access to the information is necessary, a stimulus that never reaches consciousness (e.g., an unconscious prime), would be unable to result in such a bottom-up effect. This second assumption of the GNW model is more controversial and has never been explicitly investigated. However, some indirect evidence contradicting the GNW hypothesis can be mentioned. For example, in a series of studies Bodner and Masson $(2001,2003)$ were able to demonstrate that the proportion of related trials mediates 
priming effects in a masked priming task. What is different about these studies is that they did not use an explicit target manipulation, but an implicit prime manipulation. More specifically, they manipulated the proportion of trials with a related prime. According to the GNW model it would be impossible for such an implicit context factor to exert a bottom-up impact, since this stimulus does not reach consciousness. Still, Bodner and Masson observed an increase in priming when the proportion of related versus unrelated prime trials was higher.

The present study aims to explicitly investigate the two assumptions of the GNW theory (Dehaene \& Naccache, 2001) by manipulating a context factor (the proportion of Arabic numbers versus number words) both consciously (experiment 1) and unconsciously (experiment 2). By controlling all other task conditions (task instructions, procedures, stimuli,...) and only varying whether the context factor is manipulated consciously (at the target level) or unconsciously (at the prime level), it becomes possible to directly compare the observed results for the two experiments. Based on the GNW theory we hypothesize that a fully consciously perceived context stimulus would be able to establish a reorganization of the workspace and therefore could be used strategically to enhance task performance resulting in differences in priming effects between conditions (top-down effect). Contrarily, it would be impossible for an unconscious context stimulus to establish a reorganization of the workspace and therefore it could not be used strategically to respond faster and/or more accurate to the target (bottom-up effect).

\section{Experiment 1}

In experiment 1 number targets needed to be categorized as smaller or larger than five. The targets were always preceded by novel primes (i.e. primes that were never presented as targets). This experiment was used to investigate the top-down influence of conscious stimuli by using a manipulation of the targets. We manipulated the proportion Arabic targets (e.g., "1", "4", etc.) versus number word targets (e.g., "one", "four", etc.). This manipulation created three conditions: in the first (baseline) condition subjects were presented with $50 \%$ Arabic and 50\% number word targets (condition 1a); in the second condition there were 25\% Arabic and $75 \%$ number word targets (condition 1b); and in the third condition 75\% Arabic and $25 \%$ number word targets were presented to the subjects (condition 1c). Thus, in this first experiment, only the proportion of Arabic versus number word targets was manipulated. The proportion Arabic versus number word primes was not manipulated: in each condition 50\% of the primes were Arabic numbers and 50\% were number words. Furthermore, the following variables were manipulated within subjects: prime notation (either Arabic numbers or number words), target notation (either Arabic numbers or number words) and congruency (prime and target congruent or incongruent).

Using this design, we wanted to determine whether a manipulation of the proportion of Arabic/number word targets had a top-down effect, which would be in line with previous findings (e.g., Kunde et al., 2003). The GNW theory proposes that a stimulus which is consciously perceived can establish a top-down reorganization of the workspace: a fully visual stimulus can be used in a strategic way to enhance task performance. Since in experiment 1 the targets are presented well above visual threshold and the proportion of Arabic versus number word targets is being manipulated, we expected that the subjects would process the primes differently in each condition of experiment 1 as a result of maximizing their task performance. In other words, according to the GNW theory clear differences in response patterns are expected between the three conditions of experiment 1 . More specifically, we expected that the congruency effect (i.e. faster and/or more accurate response on trials where prime and target elicit the same response as opposed to trials where prime and target evoke opposite responses) for a certain prime notation (Arabic numbers or number words) will be more prominent if the proportion of targets in that notation is large as opposed to when the proportion of targets in that notation is small. For example, in condition $1 \mathrm{~b}$ subjects receive a large proportion of number word targets $(25 \%$ Arabic and $75 \%$ number word targets). If the subjects are able to use the targets strategically to enhance their performance, we expect that in condition $1 \mathrm{~b}$ the subjects will prepare themselves more for the number word notation. This number word notation will therefore receive an advantage and will consequently elicit stronger congruency effects than the Arabic notation, which does not have this advantage. We expect the opposite pattern of results for condition 1c where $75 \%$ Arabic and 25\% number word targets are presented. 


\subsection{Methods}

\subsubsection{Participants}

Fifteen subjects participated in condition 1a and 16 subjects participated in conditions $1 \mathrm{~b}$ and $1 \mathrm{c}$. One of the subjects of condition $1 \mathrm{~b}$ was omitted from further analyses because she made an erroneous response on half of the trials. Thus, the final sample consisted of 46 subjects ( 8 male, 38 female) with a mean age of $21.61(S D=7.34$, range 17-47).

\subsubsection{Apparatus}

Stimuli were presented on a 15 -inch colour screen connected to a computer running the Windows operating system. Stimulus delivery and the recording of behavioral data (reaction time and accuracy) were controlled by E-prime (www.pstnet.com; Psychology Software Tools).

\subsubsection{Procedure}

The procedure was identical for all three conditions of the experiment, with the exception that the three separate parts differed in the proportion of Arabic versus number word targets. Each trial consisted of the following sequence. First, a forward mask was shown for $300 \mathrm{~ms}$. This mask consisted of six symbols $(\$ \# \$ \# \#)$. Then a prime stimulus was presented for $33 \mathrm{~ms}$. The prime was immediately replaced by a

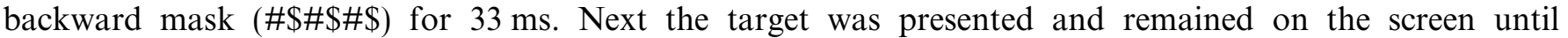
the participant had responded. The inter-trial interval was $1500 \mathrm{~ms}$. All presentations were synchronized with the vertical refresh cycle of the screen $(16.7 \mathrm{~ms})$. The forward and backward masks were shown in Arial $16 \mathrm{pt}$ and were $2.3 \mathrm{~cm}$ in width and $0.6 \mathrm{~cm}$ in height. The primes and targets appeared in Arial $14 \mathrm{pt}$. The number words width ranged from $0.8 \mathrm{~cm}$ to $1.4 \mathrm{~cm}$. The Arabic numbers were $0.3 \mathrm{~cm}$ wide. All primes and targets were $0.4 \mathrm{~cm}$ high.

\subsubsection{Stimuli}

In each condition of the experiment, the nature of the stimuli was identical. Half of the Arabic numbers and number words were only used as targets, while the other half were only used as primes. The Arabic numbers 1, 4, 6 and 9 and the Dutch number words "een" (one), "vier" (four), "zes" (six) and "negen" (nine) were used as targets. The Arabic numbers 2, 3, 7 and 8 and the Dutch number words "twee" (two), "drie" (three), "zeven" (seven) and "acht" (eight) were used as primes. All possible prime-target combinations were formed. This led to a total of 64 possible prime-target combinations (eight primes $\times$ eight targets). Each participant started with a training block of 16 trials in which each target was presented twice, without a prime. Then the experimental session started containing 4 blocks of 128 trials. Primes and targets were presented as white digits/letters on a black background. Half of the trials were congruent trials (both prime and target evoked the same response), whereas the other half were incongruent trials (prime and target evoked different responses). In condition 1a all combinations were presented to the same amount, resulting in 50\% Arabic number targets and 50\% number word targets. In condition $1 \mathrm{~b}$ trials with number word targets were presented three times as many as Arabic number targets, resulting in $25 \%$ Arabic number targets and 75\% number word targets. Finally in condition 1c, trials with Arabic number targets were presented three times as many as number word targets, resulting in $75 \%$ Arabic number targets and $25 \%$ number word targets. In all three conditions of the experiment, $50 \%$ of the presented primes were Arabic numbers and 50\% were number words. Participants were told that they would see targets, which needed to be classified in two categories. The response assignment was varied across participants: half of the subjects had to respond with a left hand key press ('a') when the target was smaller than 5 and with a right hand key press ('p') when the target was larger than 5; the other half did the experiment with the reversed response assignment. In experiments $1 \mathrm{a}$ and $1 \mathrm{~b}$ where 15 subjects participated, seven random participants had to press ' $a$ ' when the target was smaller than 5 and 'p' when the target was larger than 5; the other eight participants did the experiment with the reversed response assignment. Participants were instructed to respond as quickly as possible and to avoid mistakes as far as possible. Crucially, all participants were unaware of the purpose of the study and were told that a series of neutral visual flashes was presented to indicate the target. 


\subsubsection{Prime visibility}

Since all conditions in this experiment are very similar, the visibility of the primes was only measured for the baseline condition (condition 1a). The age range in this condition was 17-20 years. Prime visibility was assessed for each of the 15 participants in this baseline condition using an objective visibility test. Immediately after completing the baseline experiment, the participants were informed about the presence of the primes. They were asked to participate in a post-test, consisting of 64 trials: the same prime-target combinations used in experiment 1 were presented once. The participants were instructed to apply the same instructions they received before (cfr. experiment 1) to the prime instead of the target. If they were unable to categorize the prime, they were forced to guess (forced choice). Presentation conditions were the same as during the experiment. Analyses of the post-test revealed that although the participants were fully informed about the primes, on average they could only classify $46 \%$ of the primes correctly, which immediately indicates that primes could not be classified above chance.

\subsection{Results}

\subsection{1. $R T$ and error rate analysis}

Wrong responses (on average 4.2\%) were discarded from RT analyses. For each of the three conditions of this experiment, median RTs from correct responses and mean error rates were then submitted to a repeated measures analysis with prime notation (two levels: Arabic numbers and number words), target notation (two levels: Arabic numbers and number words) and response congruency (two levels: congruent and incongruent) as within-subject factors and target manipulation (three levels: condition 1a: 50\% Arabic and 50\% number words; condition 1b:25\% Arabic and 75\% number words; condition 1c: $75 \%$ Arabic and $25 \%$ number words) as between-subject factor. Since we want to investigate whether the congruency effect for a certain prime notation is more prominent if the proportion of targets in that notation is larger, the crucial interaction under investigation here is the interaction between prime notation $\times$ response congruency $\times$ target manipulation. Median RTs and mean error rates as a function of prime notation, target notation and congruency for conditions 1a, $1 \mathrm{~b}$ and $1 \mathrm{c}$ are listed in Tables 1 and 2.

\subsubsection{RT analyses}

For experiment 1 , the following pattern of results was observed for the median RTs. First, a significant main effect of target notation was found $(F(1,43)=108.31, p<.001)$, with number word targets on average

Table 1

Median RTs (SD) as a function of prime notation, target notation and congruency for conditions $1 \mathrm{a}, 1 \mathrm{~b}$ and $1 \mathrm{c}$

\begin{tabular}{|c|c|c|c|c|c|}
\hline & Prime notation & Target notation & Congruent & Incongruent & Difference \\
\hline \multirow[t]{4}{*}{ Condition 1a } & Arabic & Arabic & $478(37.1)$ & $482(37.7)$ & 4 \\
\hline & Arabic & Number word & 499 (37.5) & 513 (39.9) & 14 \\
\hline & Number word & Arabic & $484(30.5)$ & 497 (27.4) & 13 \\
\hline & Number word & Number word & $490(33.3)$ & $506(34.7)$ & 16 \\
\hline \multirow[t]{4}{*}{ Condition $1 \mathrm{~b}$} & Arabic & Arabic & 488 (39.6) & $497(52.0)$ & 9 \\
\hline & Arabic & Number word & $512(56.6)$ & $513(48.0)$ & 1 \\
\hline & Number word & Arabic & $501(62.7)$ & $511(66.5)$ & 10 \\
\hline & Number word & Number word & $491(54.3)$ & $516(50.4)$ & 25 \\
\hline \multirow[t]{4}{*}{ Condition 1c } & Arabic & Arabic & $504(60.3)$ & $512(55.0)$ & 8 \\
\hline & Arabic & Number word & $537(69.2)$ & $550(75.0)$ & 13 \\
\hline & Number word & Arabic & $505(59.1)$ & $513(58.6)$ & 8 \\
\hline & Number word & Number word & 549 (69.7) & $540(71.9)$ & 9 \\
\hline \multirow[t]{4}{*}{ Total } & Arabic & Arabic & $490(47.5)$ & 497 (49.5) & 7 \\
\hline & Arabic & Number word & $517(57.4)$ & $526(58.4)$ & -9 \\
\hline & Number word & Arabic & 497 (52.6) & 507 (52.9) & 10 \\
\hline & Number word & Number word & $511(60.5)$ & $521(55.6)$ & 10 \\
\hline
\end{tabular}

Note: The differences are calculated as the difference between incongruent and congruent trials (incongruent-congruent). Condition 1a: 50\% Arabic, 50\% number word targets; condition 1b: 25\% Arabic, 75\% number word targets; condition 1c: $75 \%$ Arabic, 25\% number word targets. 
Table 2

Mean error rates (SD) as a function of prime notation, target notation and congruency for conditions $1 \mathrm{a}, 1 \mathrm{~b}$ and $1 \mathrm{c}$

\begin{tabular}{|c|c|c|c|c|c|}
\hline & Prime notation & Target notation & Congruent & Incongruent & Difference \\
\hline \multirow[t]{4}{*}{ Condition 1a } & Arabic & Arabic & $3.4(2.4)$ & $2.9(2.8)$ & -0.5 \\
\hline & Arabic & Number word & $3.6(2.7)$ & $4.1(2.1)$ & 0.5 \\
\hline & Number word & Arabic & $2.8(1.9)$ & $3.7(3.9)$ & 0.9 \\
\hline & Number word & Number word & $3.7(2.8)$ & $4.6(3.3)$ & 0.9 \\
\hline \multirow[t]{4}{*}{ Condition $1 \mathrm{~b}$} & Arabic & Arabic & $3.1(3.3)$ & $3.7(3.9)$ & 0.6 \\
\hline & Arabic & Number word & $4.7(4.1)$ & $6.1(4.7)$ & 1.4 \\
\hline & Number word & Arabic & $4.2(4.7)$ & $4.6(5.8)$ & 0.4 \\
\hline & Number word & Number word & $4.1(4.1)$ & $6.2(5.7)$ & 2.1 \\
\hline \multirow[t]{4}{*}{ Condition $1 \mathrm{c}$} & Arabic & Arabic & $3.3(3.0)$ & $4.0(4.0)$ & 0.7 \\
\hline & Arabic & Number word & $4.1(5.3)$ & $7.8(12.3)$ & 3.7 \\
\hline & Number word & Arabic & $3.8(3.0)$ & $3.4(2.4)$ & -0.4 \\
\hline & Number word & Number word & $4.9(4.1)$ & $4.7(4.3)$ & -0.2 \\
\hline \multirow[t]{4}{*}{ Total } & Arabic & Arabic & $3.3(2.9)$ & $3.6(3.6)$ & 0.3 \\
\hline & Arabic & Number word & $4.1(4.1)$ & $6.0(7.8)$ & 1.9 \\
\hline & Number word & Arabic & $3.6(3.3)$ & $3.9(4.2)$ & 0.3 \\
\hline & Number word & Number word & $4.2(3.7)$ & $5.1(4.5)$ & 0.9 \\
\hline
\end{tabular}

Note: The differences are calculated as the difference between incongruent and congruent trials (incongruent-congruent). Condition 1a: 50\% Arabic, 50\% number word targets; condition 1b: 25\% Arabic, 75\% number word targets; condition 1c: 75\% Arabic, 25\% number word targets.

being responded to $21 \mathrm{~ms}$ slower than Arabic targets. This main effect can be interpreted in interaction with prime notation $(F(1,43)=16.79, p<.001)$. Targets were responded to faster when they were preceded by a prime with the same notation (e.g., Arabic prime, Arabic target). We also found a significant interaction between prime notation, target notation and target manipulation $(F(2,43)=4.02, p=.03)$. In conditions $1 \mathrm{a}$ and $1 \mathrm{~b}$, this same-notation advantage was clearly present, whereas it was absent in condition $1 \mathrm{c}$. The effect of target notation also interacted with the target manipulation $(F(2,43)=16.37, p<.001)$. This interaction effect indicates that subjects in all conditions responded slower when the target was a number word as opposed to an Arabic number. However, this difference in RT between Arabic and number word targets was more evident when more Arabic numbers were presented (condition 1c versus conditions 1a and 1b): respectively $36 \mathrm{~ms}, 17 \mathrm{~ms}$ and $9 \mathrm{~ms}$. Furthermore, a significant main effect of congruency was observed $(F(1$, $43)=25.27, p<.001$ ) with congruent trials on average being responded $9 \mathrm{~ms}$ faster than incongruent trials. However, this main effect again interacted with other effects. Crucially, we observed a significant interaction between prime notation, congruency and target manipulation $(F(2,43)=3.70, p=.03)$. For condition $1 \mathrm{a}$, where an equal amount of Arabic and number word targets was presented, the congruency effect was apparent for both the number word primes $(15 \mathrm{~ms})$ and the Arabic primes $(9 \mathrm{~ms})$. Contrarily, for condition $1 \mathrm{~b}$, where more number word targets were presented, the congruency effect was more pronounced for number word primes $(18 \mathrm{~ms})$ than for Arabic primes $(5 \mathrm{~ms})$. In condition 1c, where more Arabic targets were presented, we found a reversed effect: here the congruency effect was more pronounced for Arabic primes (11 ms) as compared to number word primes $(0 \mathrm{~ms})$. This interaction under investigation is also depicted in Fig. 1. Finally, a significant four-way interaction was present between prime notation, target notation, congruency and target manipulation $(F(2,43)=4.38, p=.02)$. The direction of this four-way interaction is shown in Table 1 . The effect of the between-subject factor, the target manipulation, was not significant $(F(2,43)=1.68, p=.20)$. None of the other effects were significant.

\subsubsection{Error analyses}

The following pattern of results was observed for the mean error rates. First, a significant main effect of target notation was found $(F(1,43)=11.43, p=.002)$. More errors were made on number word targets as compared to Arabic targets (4.9\% versus 3.6\%). Second, a significant main effect of congruency was found $(F(1,43)=5.05, p=.03)$. On average, subjects made more errors on incongruent trials as compared to 


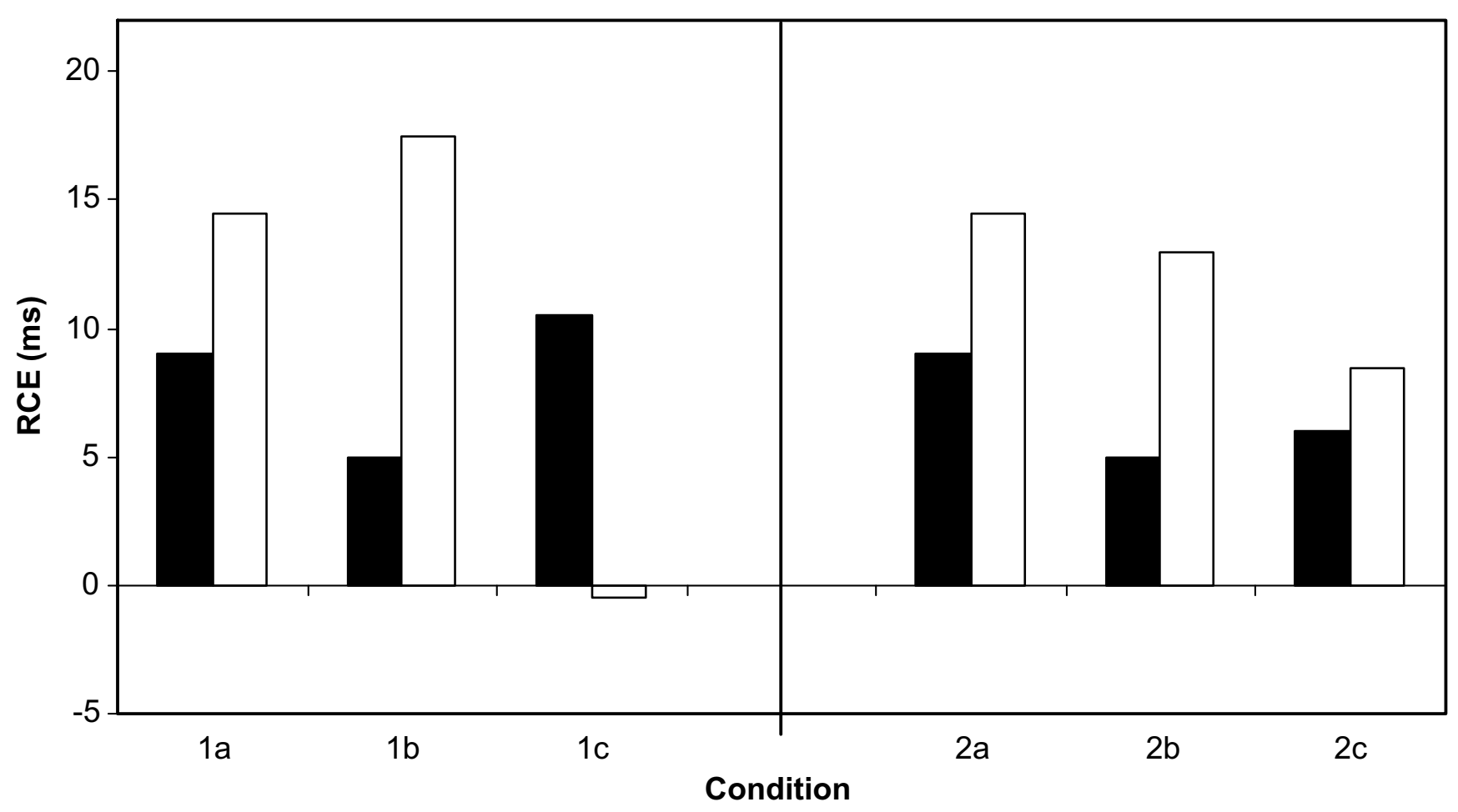

- Arabic primes $\square$ Number word primes

Fig. 1. The differences (RCEs) between congruent and incongruent trials (incongruent-congruent) as a function of prime notation (Arabic or number word) for the three conditions of experiment 1 (target manipulation, left panel) and 2 (prime manipulation, right panel). Condition 1a: 50\% Arabic targets, 50\% number word targets; condition 1b: $25 \%$ Arabic targets, $75 \%$ number word targets; condition 1c: $75 \%$ Arabic targets, $25 \%$ number word targets; condition 2a: 50\% Arabic primes, 50\% number word primes; condition 2b: $25 \%$ Arabic primes, $75 \%$ number word primes; condition $2 \mathrm{c}$ : $75 \%$ Arabic primes, $25 \%$ number word primes.

congruent trials $(4.7 \%$ versus $3.8 \%)$. The interaction between prime notation, congruency and target manipulation was not significant $(F(2,43)=1.96, p=.15)$. The effect of the between-subject factor, the target manipulation, was not significant $(F(2,43)=0.52, p=.60)$. None of the other effects were significant.

\subsection{Discussion}

In experiment 1 , the crucial interaction under investigation, namely the interaction between prime notation, congruency and target manipulation, was significant in the RT analyses. This indicates that the congruency effect for a certain prime notation (Arabic numbers or number words) was more prominent if the proportion of targets in that notation was large as opposed to when the proportion of targets in that notation was small. For condition $1 \mathrm{~b}$, where the proportion of number word targets was large, there was a stronger congruency effect present for the number word primes compared to condition 1c, where the proportion of number word targets was small. Equivalently, for condition 1c, where the proportion of Arabic targets was large, a stronger congruency effect was observed for the Arabic primes as compared to condition $1 \mathrm{~b}$, where the proportion of Arabic targets was small.

This is completely in line with the GNW theory: the manipulation of the proportion of targets in Arabic versus number word notation has an influence on the observed congruency effects. Thus, in line with previous research findings (e.g., Greenwald et al., 2003; Kunde et al., 2003), top-down influencing of the workspace indeed seems possible. Kunde et al. (2003) assume that subjects can create intentions based on the stimulus characteristics (formation of action trigger sets) and apply these intentions to the processing of the primes. This could be an explanation for the results obtained in experiment 1 . The significant interaction between prime notation $\times$ congruency $\times$ target manipulation could indicate that subjects form their action triggers 
based on the targets they receive. When a certain target format is presented more frequently, the action trigger set will be more biased in favour of this notation, and the chance is larger that a match will be reached with primes in that notation, leading to stronger priming effects for that notation.

At first sight, these results seem to contradict the cross-notational priming observed by Van Opstal, Reynvoet, and Verguts (2005). They found significant congruency effects not only for primes presented in the same notation as the targets, but also for primes that were presented in a different notation. However, the priming effects for primes in a different notation were clearly less apparent than the priming effects for primes in the same notation as the targets, which is in line with our present findings. The use of a more complex, and therefore more efficient mask (i.e. $\$ \# \$ \$ \#$ as forward mask and $\# \$ \# \# \$$ as backward mask), could explain why the cross-notational priming (but also the within-notational priming) is less prominent in the present experiment.

We also note that the proportion manipulation in experiment 1 affected both the impact of number word primes and Arabic primes. For example, on trials where both prime and target are number words, priming is more evident when $75 \%$ number word targets are shown $(25 \mathrm{~ms})$ compared to when only $25 \%$ number word targets are shown $(-9 \mathrm{~ms}) .{ }^{1}$ Contrarily, on trials where the prime is an Arabic number but the target is a number word, priming is clearly more evident when $75 \%$ Arabic targets are shown (13 ms) compared to when only $25 \%$ Arabic targets are shown $(1 \mathrm{~ms})$. Even though the example above shows that the influence of both prime notations was affected by the target manipulation, this effect was somewhat larger for number word primes. This could be due to the fact that Arabic numbers and number words need to be processed differently (Fias, 2001; Reynvoet \& Brysbaert, 2004). The meaning of Arabic numbers is reached by a one-step symbol translation, whereas more processing steps are required for number words (letter identification, letter combination, word identification, ...). It could be that the latter process is more susceptible to the notation manipulation resulting in a larger effect for number words.

\section{Experiment 2}

The second experiment was designed to investigate the bottom-up influence of unconscious stimuli by using a prime manipulation. Experiment 2 resembles experiment 1 very closely. However, in this experiment, the between-subject manipulation was altered. Now, we manipulated the proportion of Arabic primes (e.g., "2", "3", etc.) versus number word primes (e.g., "two", "three", etc.) between subjects. This manipulation again created three conditions: in the first (baseline) condition subjects were presented with 50\% Arabic and $50 \%$ number word primes (condition $2 \mathrm{a}$ ); in the second condition there were $25 \%$ Arabic and $75 \%$ number word primes (condition $2 \mathrm{~b}$ ); and in the third condition 75\% Arabic and $25 \%$ number word primes were presented to the subjects (condition 2c). Thus, in this second experiment, only the proportion of Arabic versus number word primes was manipulated. The proportion Arabic versus number word targets was not manipulated: in each condition 50\% of the targets were Arabic numbers and 50\% were number words.

Using this design, we wanted to determine whether this manipulation of the proportion of Arabic/number word primes had an effect on the prime processing. According to the GNW theory, strategic use of the prime would require conscious perception of the prime. Reasoning along these lines, if a subject in experiment 2 would want to use the manipulation of the proportion of Arabic/number word primes strategically to enhance his/her performance on the task, he/she should be consciously aware of the prime. Since this is not the case in experiment 2 (the primes are presented very shortly and are masked), we expected that the manipulation of the proportion of Arabic/number word primes would have no effect. In other words, according to the GNW theory no differences in response patterns are expected between the three conditions of experiment 2 . However, if our results do not support the GNW we would expect that the congruency effect for a certain prime notation (Arabic numbers or number words) will be more prominent if the proportion of primes in that notation is large as opposed to when the proportion of primes in that notation is small. For example, in condition $2 \mathrm{~b}$ subjects receive a large proportion of number word primes (25\% Arabic and $75 \%$ number word primes). If the subjects are able to use the primes strategically to enhance their performance, we expect that in condition

\footnotetext{
${ }^{1}$ A paired samples $t$-test showed that no significant difference was observed between reaction times on the congruent trials and incongruent trials for experiment 1 when both prime and target were presented as number words $(\mathrm{RCE}=-9 \mathrm{~ms}, t(15)=1.80, p=.09)$.
} 
$2 \mathrm{~b}$ the subjects will prepare themselves more for the number word notation. This number word notation will therefore receive a strategic advantage when presented as primes and will consequently elicit stronger congruency effects than the Arabic primes, which do not have this strategic advantage. We expect the opposite pattern of results for condition 2c, where 75\% Arabic and 25\% number word primes are presented.

\subsection{Method}

\subsubsection{Participants}

Forty-seven subjects participated in the experiment (9 male, 38 female) with a mean age of 19.75 $(S D=2.65$, range 17-30). Since condition $2 \mathrm{a}$ is identical to condition 1a, the results of these 15 subjects were reused for experiment 2 . In both conditions $2 \mathrm{~b}$ and $2 \mathrm{c} 16$ subjects participated, none of which had participated in experiment 1.

\subsubsection{Apparatus, stimuli and procedure}

Apparatus, stimuli and procedure were exactly the same as in Experiment 1. However, the between-subject manipulation changed. Now, the proportion of Arabic versus number word primes was manipulated instead of the targets. Condition 2a was identical to condition 1a: $50 \%$ of the presented primes were Arabic numbers and $50 \%$ were number words. In condition $2 \mathrm{~b}$ trials with number word primes were presented three times as many as Arabic number primes, resulting in 25\% Arabic number primes and $75 \%$ number word primes. Finally in condition 2c, trials with Arabic number primes were presented three times as many as number word primes, resulting in 75\% Arabic number primes and 25\% number word primes. In all three conditions of the experiment, $50 \%$ of the presented targets were Arabic numbers and 50\% were number words.

\subsubsection{Prime visibility}

Since experiment 2 is very similar to experiment 1 , and the prime visibility for this first experiment was clearly below chance level, the visibility of the primes was not measured again for experiment 2 .

\subsection{Results}

\subsection{1. $R T$ and error rate analysis}

Wrong responses (on average 4.4\%) were discarded from RT analyses. For each of the three conditions of this experiment, median RTs from correct responses and mean error rates were then submitted to a repeated measures analysis with prime notation (two levels: Arabic numbers and number words), target notation (two levels: Arabic numbers and number words) and response congruency (two levels: congruent and incongruent) as within-subject factors and prime manipulation (three levels: condition 2a: 50\% Arabic, 50\% number words; condition 2b: 25\% Arabic, 75\% number words; condition 2c: 75\% Arabic, 25\% number words) as betweensubject factor. Since we want to investigate whether the congruency effect for a certain prime notation is more prominent if the proportion of primes in that notation is larger, the crucial interaction under investigation here is the interaction between prime notation $\times$ response congruency $\times$ prime manipulation (note that the interaction between prime notation and prime manipulation would not be informative, since the subjects have to respond to the target). Median RTs and mean error rates as a function of prime notation, target notation and congruency for conditions $2 \mathrm{a}, 2 \mathrm{~b}$ and $2 \mathrm{c}$ are listed in Tables 3 and 4.

\subsubsection{RT analyses}

For experiment 2, the following pattern of results was observed for the median RTs. First, a significant main effect of target notation was found $(F(1,44)=97.59, p<.001)$, with number word targets on average being responded to $18 \mathrm{~ms}$ slower than Arabic targets. This main effect can be interpreted in interaction with prime notation $(F(1,44)=24.55, p<.001)$. Targets were responded to faster when they were preceded by a prime with the same notation (e.g., Arabic prime, Arabic target). The main effect of prime manipulation was not significant $(F(2,44)=0.64, p=.53)$ and did not interact with either target notation, or prime notation $(F(2,44)=1.33, p=.28 ; F(2,44)=0.97, p=.39$ respectively $)$. A significant main effect of congruency was observed $(F(1,44)=51.12, p<.001)$ with congruent trials on average being responded $9 \mathrm{~ms}$ faster than incon- 
Table 3

Median RTs (SD) as a function of prime notation, target notation and congruency for conditions $2 \mathrm{a}, 2 \mathrm{~b}$ and $2 \mathrm{c}$

\begin{tabular}{|c|c|c|c|c|c|}
\hline & Prime notation & Target notation & Congruent & Incongruent & Difference \\
\hline \multirow[t]{4}{*}{ Condition $2 \mathrm{a}$} & Arabic & Arabic & $478(37.1)$ & $482(37.7)$ & 4 \\
\hline & Arabic & Number word & $499(37.5)$ & $513(39.9)$ & 14 \\
\hline & Number word & Arabic & $484(30.5)$ & 497 (27.4) & 13 \\
\hline & Number word & Number word & $490(33.3)$ & $506(34.7)$ & 16 \\
\hline \multirow[t]{4}{*}{ Condition 2b } & Arabic & Arabic & $502(66.1)$ & $504(67.2)$ & 2 \\
\hline & Arabic & Number word & $521(59.1)$ & $529(58.2)$ & 8 \\
\hline & Number word & Arabic & $502(65.4)$ & $510(59.3)$ & 8 \\
\hline & Number word & Number word & $505(59.7)$ & $523(58.2)$ & 18 \\
\hline \multirow[t]{4}{*}{ Condition $2 \mathrm{c}$} & Arabic & Arabic & $481(40.7)$ & 491 (40.6) & 10 \\
\hline & Arabic & Number word & $511(43.3)$ & $513(47.9)$ & 2 \\
\hline & Number word & Arabic & $486(37.8)$ & 498 (42.7) & 12 \\
\hline & Number word & Number word & $507(51.4)$ & $512(50.0)$ & 5 \\
\hline \multirow[t]{4}{*}{ Total } & Arabic & Arabic & $487(50.0)$ & $492(50.3)$ & 5 \\
\hline & Arabic & Number word & $511(47.5)$ & $518(49.0)$ & 7 \\
\hline & Number word & Arabic & $491(47.0)$ & $502(44.8)$ & 11 \\
\hline & Number word & Number word & $501(49.2)$ & $514(48.3)$ & 13 \\
\hline
\end{tabular}

Note: The differences are calculated as the difference between incongruent and congruent trials (incongruent-congruent). Condition $2 \mathrm{a}$ : 50\% Arabic, 50\% number word primes; condition 2b: 25\% Arabic, $75 \%$ number word primes; condition 2c: $75 \%$ Arabic, $25 \%$ number word primes.

Table 4

Mean error rates (SD) as a function of prime notation, target notation and congruency for conditions $2 \mathrm{a}, 2 \mathrm{~b}$ and $2 \mathrm{c}$

\begin{tabular}{|c|c|c|c|c|c|}
\hline & Prime notation & Target notation & Congruent & Incongruent & Difference \\
\hline \multirow[t]{4}{*}{ Condition $2 \mathrm{a}$} & Arabic & Arabic & $3.4(2.4)$ & $2.9(2.8)$ & -0.5 \\
\hline & Arabic & Number word & $3.6(2.7)$ & $4.1(2.1)$ & 0.5 \\
\hline & Number word & Arabic & $2.8(1.9)$ & $3.7(3.9)$ & 0.9 \\
\hline & Number word & Number word & $3.7(2.8)$ & $4.6(3.3)$ & 0.9 \\
\hline \multirow[t]{4}{*}{ Condition $2 b$} & Arabic & Arabic & $3.5(3.9)$ & $2.5(2.3)$ & -1.0 \\
\hline & Arabic & Number word & $3.7(4.1)$ & $4.3(4.7)$ & 0.6 \\
\hline & Number word & Arabic & $2.5(1.9)$ & $3.1(3.2)$ & 0.6 \\
\hline & Number word & Number word & $3.4(2.7)$ & $5.4(4.4)$ & 2.0 \\
\hline \multirow[t]{4}{*}{ Condition 2c } & Arabic & Arabic & $4.3(3.1)$ & $6.0(4.9)$ & 1.7 \\
\hline & Arabic & Number word & $7.0(5.6)$ & $7.3(4.7)$ & 0.3 \\
\hline & Number word & Arabic & $3.5(4.4)$ & $6.2(3.9)$ & 2.7 \\
\hline & Number word & Number word & $6.4(6.2)$ & $6.2(5.6)$ & -0.2 \\
\hline \multirow[t]{4}{*}{ Total } & Arabic & Arabic & $3.7(3.2)$ & $3.8(3.8)$ & 0.1 \\
\hline & Arabic & Number word & $4.8(4.5)$ & $5.2(4.2)$ & 0.4 \\
\hline & Number word & Arabic & $2.9(3.0)$ & $4.4(3.8)$ & 1.5 \\
\hline & Number word & Number word & $4.5(4.4)$ & $5.4(4.5)$ & 0.9 \\
\hline
\end{tabular}

Note: The differences are calculated as the difference between incongruent and congruent trials (incongruent-congruent). Condition 2a: 50\% Arabic, 50\% number word primes; condition 2b: 25\% Arabic, $75 \%$ number word primes; condition 2c: $75 \%$ Arabic, $25 \%$ number word primes.

gruent trials. Crucially, the interaction between prime notation, congruency and prime manipulation was not significant $(F(2,44)=0.22, p=.81)$. The results indicate that the congruency effect was more apparent for number word primes than for Arabic primes, for all conditions. This interaction under investigation is also depicted in Fig. 1. However, the interaction between target notation, congruency and prime manipulation was marginally significant $(F(2,44)=3.18, p=.05)$. For both conditions $2 \mathrm{a}$ and $2 \mathrm{~b}$ the congruency effect was most evident for the number word targets (respectively $15 \mathrm{~ms}$ and $13 \mathrm{~ms}$ ) and less apparent for the Arabic targets (respectively $9 \mathrm{~ms}$ and $5 \mathrm{~ms}$ ). However, in condition $2 \mathrm{c}$ we found a reversed effect: here the congruency 
effect was more pronounced for Arabic targets $(11 \mathrm{~ms})$ as compared to number word targets $(4 \mathrm{~ms})$. None of the other effects were significant.

\subsubsection{Error analyses}

The following pattern of results was observed for the mean error rates. First, a significant main effect of target notation was found $(F(1,44)=15.45, p<.001)$. More errors were made on number word targets as compared to Arabic targets (5.0\% versus 3.7\%). Second, a marginally significant main effect of congruency was observed $(F(1,44)=3.45, p=.07)$. On average, subjects made more errors on incongruent trials as compared to congruent trials $(4.7 \%$ versus $4.0 \%)$. Furthermore, congruency interacted with prime notation $(F(1$, $44)=4.25, p=.04)$ : the congruency effect was more prominent for number word primes than for Arabic primes. As in the RT analyses, the interaction between prime notation, congruency and prime manipulation was not significant $(F(2,44)=0.93, p=.40)$. The results show that the congruency effect was more pronounced when the prime was presented in number word notation as compared to Arabic notation for all three conditions of the experiment. However, a significant interaction between target notation, congruency and prime manipulation was present $(F(2,44)=3.83, p=.03)$. For both conditions $2 \mathrm{a}$ and $2 \mathrm{~b}$ the congruency effect was more evident for number word targets as compared to Arabic targets, whereas for condition $2 \mathrm{c}$ a reversed effect was observed: here the congruency effect was more pronounced for Arabic targets as compared to number word targets. The effect of the between-subject factor, the prime manipulation, reached significance $(F(2,44)=3.57, p=.04)$. None of the other effects were significant.

\subsection{Discussion}

Most interestingly, the crucial interaction under investigation, namely the interaction between prime notation, congruency and prime manipulation, was not significant. This indicates that the congruency effect for a certain prime notation (Arabic numbers or number words) was not more prominent if the proportion of primes in that notation was large as opposed to when the proportion of primes in that notation was small. For condition $2 b$, where the proportion of number word primes was large, there was no stronger congruency effect present for the number word primes compared to condition $2 \mathrm{c}$, where the proportion of number word primes was small. Equivalently, for condition 2c, where the proportion of Arabic primes was large, no stronger congruency effect was observed for these Arabic primes as compared to condition $2 \mathrm{~b}$, where the proportion of Arabic primes was small. This is completely in line with the GNW theory and indicates that a manipulation of unconscious primes indeed does not result in intentions in favor of one of the prime notations.

However, contrary to our expectations, a marginally significant interaction was observed between target notation, congruency and prime manipulation. At this point, we find it difficult to provide an explanation for this unexpected effect. It appears that the congruency effect for a certain target notation (Arabic numbers or number words) is more apparent if the proportion of primes in that notation was large as opposed to when the proportion of primes in that notation was small.

A possible explanation might be that presenting a higher proportion of a certain notation as primes may lead to a bottom-up effect dependent upon very low level processes such as letter identification and word construction for number words and symbol translation for Arabic numbers. This bottom-up effect would then result in a faster (since repeated) identification of that stimulus notation that is presented the most as primes and should therefore lead to faster RTs for that notation. However, when a stimulus notation is presented repeatedly, not only will it be identified faster, but the subsequent steps in the processing chain of categorisation and response activation will also be faster. In other words, if the target notation matches the highest proportion ("preferred") prime notation, the target will be processed faster through the processing chain and will activate more rapidly its corresponding motor response leading to a larger response congruency effect. Because this effect results in faster RTs on congruent trials and slower RTs on incongruent trials, the prime manipulation $\times$ target notation interaction will not reach significance because both type of trials neutralize each other. However, this does result in larger congruency effects for those trials where the target has the same notation as the most frequent prime notation (i.e. marginally significant prime manipulation $\times$ target notation $\times$ congruency interaction). 
This explanation predicts larger congruency effects for those trials where the target has the same notation as the most frequent prime notation: for condition $2 \mathrm{~b}(75 \%$ number word primes) we would expect stronger effects for trials where the target is a number word; for condition $2 \mathrm{c}$ ( $75 \%$ Arabic primes) we would expect stronger effects for trials where the target is an Arabic number. This also means that the pattern of results for experiments 1 and 2 would differ with regard to the cross-notation trials (number word prime - Arabic target trials and Arabic prime - number word target trials), but not with regard to the same-notation trials (both prime and target number words or both Arabic numbers). This is exactly what we observe. We want to stress that this alternative explanation for experiment 2 is not necessarily against the GNW model: due to mere "lower-level" bottom-up strength of the unconscious primes (due to repeated presentation of the highest proportion prime notation), they could have been able to result some effect. However, the bottomup strength is clearly too limited to evoke the use of strategies from the subjects, as can be seen form the non-significant interaction between prime notation $\times$ congruency $\times$ prime manipulation.

It should also be noted that this three-way interaction is only marginally significant in the RT analyses $(p=.05)$ and that the results of the error analyses altogether are very weak, given the small error rates for the task at hand. Furthermore, this marginal significant interaction seems to stem from the highly significant main effects of target notation and congruency (both $p<.001$ ), since all the two-way interactions between these three factors (target notation, congruency and prime manipulation) are not significant. Therefore, it could also be hypothesized that this counterintuitive effect reflects a spurious effect rather than reflecting a genuine bottom-up effect from the prime. However, further research seems warranted to clarify this issue.

\section{General discussion}

The goal of this study was to clarify whether a conscious (target) and unconscious (prime) manipulation of the proportion of Arabic numbers versus number words had an effect on prime processing. Regarding this issue, the global neuronal workspace theory (Dehaene \& Naccache, 2001) made two basic assumptions. First, according to this model, it would be impossible for an unconscious stimulus to be used strategically to enhance task performance (bottom-up effect). Contrarily, a fully consciously perceived stimulus would have the ability to be used strategically to enhance task performance (top-down effect). This latter assumption has recently received a lot of attention, and the observed research results support the GNW theory (e.g., Kunde et al., 2003). However, the former hypothesis has not entered the spotlight yet and has never been directly investigated, although some indirect evidence can be found contradicting the GNW assumption (e.g., Bodner \& Masson, 2003).

In a first experiment we examined whether conscious stimuli can establish a reorganization of the workspace (top-down effect). This hypothesis was investigated by introducing a manipulation of a conscious context factor, namely the proportion of Arabic versus number word targets, creating three conditions $(50 \%$ Arabic targets-50\% number word targets, $25 \%-75 \%$ and $75 \%-25 \%$ ). If the conscious (target) manipulation would have resulted in a top-down effect, we should have found a differential response pattern depending on the target manipulation: if the subjects would have been able to use the target manipulation to enhance their task performance, they would have prepared themselves more for the notation that appeared as targets on $75 \%$ of the trials. Consequently, this notation would have received a strategic advantage and would therefore have elicited stronger congruency effects than the notation that was presented as targets on only $25 \%$ of the trials. Indeed, the results indicated that the observed response pattern was different for the three conditions, depending on the manipulation of the proportion Arabic/number word targets: the congruency effect for a certain prime notation (Arabic numbers or number words) was more prominent if the proportion of targets in that notation was large as opposed to when the proportion of targets in that notation was small. These results provide clear evidence in favor of the GNW theory and indicate that the conscious manipulation has an influence on the observed congruency effects. Thus, in line with previous research results, top-down influencing of the workspace by conscious context factors is possible.

A second experiment was designed to clarify whether unconscious stimuli can nevertheless elicit a reorganization of the workspace (bottom-up effect). This hypothesis was investigated using the same experimental conditions as in the first experiment, but now an unconscious context factor was manipulated, namely the proportion of Arabic versus number word primes, again creating three conditions (50\% Arabic primes-50\% num- 
ber word primes, $25 \%-75 \%$ and $75 \%-25 \%$ ). If the unconscious (prime) manipulation would have resulted in a bottom-up effect, we should have found a differential response pattern depending on the prime manipulation: if the subjects would have been able to use the prime manipulation to enhance their task performance, they would have prepared themselves more for the notation that appeared as primes on $75 \%$ of the trials. Consequently, this notation would have received a strategic advantage and would therefore have elicited stronger congruency effects than the notation that was presented as primes on only $25 \%$ of the trials. However, the results indicated that the observed response pattern was similar in all three conditions, regardless of the manipulation of the proportion Arabic/number word primes. Again, this is in line with the GNW theory and indicates that the unconscious manipulation indeed did not result in a bottom-up effect.

The observed patterns of results were clearly different for experiment 1 and experiment 2 (see also Fig. 1). In experiment 1 the congruency effect for a certain prime notation (Arabic numbers or number words) was more pronounced if the proportion of targets in that notation was large. When the target set consisted of $75 \%$ number word targets, the congruency effect was stronger for the number word primes (condition 1b); when more Arabic numbers were presented as targets, the congruency effect was stronger for the Arabic primes (condition 1c). In experiment 2, a different pattern emerged: the congruency effect for a certain target notation (Arabic numbers or number words) was more evident if the proportion of primes in that notation was large. For condition $2 \mathrm{~b}(75 \%$ number word primes) the congruency effect was stronger when the target was a number word; for condition 2c (75\% Arabic primes) the congruency effect was larger for Arabic targets. This leads to a similar pattern of results for experiments 1 and 2 for the same-notation trials (both prime and target are number words or both are Arabic numbers), but to a completely opposite pattern of results for both experiments for the cross-notation trials (Arabic prime and number word target or number word prime and Arabic target).

This indicates that fundamentally different processes have to lie at the basis of the observed data patterns for experiments 1 and 2. In experiment 1 top-down strategies were used by the subjects to enhance performance (significant prime notation $\times$ congruency $\times$ prime manipulation interaction). In experiment 2 subjects were not able to form such strategies (no significant prime notation $\times$ congruency $\times$ prime manipulation interaction). However, it could be that presenting a certain stimulus notation more frequently as unconscious primes results in a low-level processing advantage (as argued in the discussion of experiment 2) that might have had some effect (marginally significant target notation $\times$ congruency $\times$ prime manipulation interaction). However, it also seems plausible that this interaction reflects a spurious effect.

How can we reconcile our present finding with the studies of Bodner and Masson $(2001,2003)$ which did obtain evidence for the creation of intentions based on an unconscious (prime) manipulation? Bodner and Masson manipulated the proportion of related versus unrelated prime-target pairs by manipulating the masked prime validity (relatedness proportion, RP). In line with the GNW hypotheses, such unconscious manipulations are unlikely to allow subjects to form conscious, strategic intentions that can aid them to use the primes to enhance task performance. Still, such masked prime-validity effects have been obtained (e.g., Bodner \& Dypvik, 2005; Bodner \& Masson, 2001, 2003), although not under all conditions (e.g., Perea \& Rosa, 2002). A possible, yet speculative explanation for these masked prime-validity effects might lie in the nature of the prime manipulation. Suppose a subject is presented with a condition where only $20 \%$ of the primes are valid for the target and $80 \%$ of the trials are unrelated trials (.20 RP condition). In such a situation, the subject could get the impression that he/she always tends to give the wrong answer: the prime and target are incongruent (i.e. leading to opposite responses) on $80 \%$ of the trials, which means that the prime leads to a wrong answer in $80 \%$ of the cases. When the subject realizes this (consciously or unconsciously), he/she could decide to completely ignore the stimulation before and during the prime, since they lead to the wrong decision most of the time. Therefore, in such a condition with only $20 \%$ related trials, the priming effects would be strongly reduced as compared to a condition with $80 \%$ related prime-target pairs (.80 RP condition). Reasoning along these lines, the discrepancy between the (stronger) priming effects in the $.80 \mathrm{RP}$ condition and the (smaller) priming effects in the $.20 \mathrm{RP}$ condition would be due to the ignoring of the primes in the .20 condition by the subjects and not to a differential strategic use of the unconscious prime manipulation. Bodner and Dypvik (2005) made a similar argument in the form of the differential attention account. According to this theory, the prime validity might influence how much attention is dedicated to the masked primes. If this is the case, subjects in the $.20 \mathrm{RP}$ condition simply learn to pay less attention to the primes than subjects in the $.80 \mathrm{RP}$ condition, which would explain the weak priming effects in the $.20 \mathrm{RP}$ condition. Although 
speculative, this theory would explain why we did not find a more prominent congruency effect for a certain prime notation if the proportion of primes in that notation was large. In our experiment, the number of related and unrelated trials was always identical (50\%), so in all conditions of the present experiments $50 \%$ of the primes were congruent (i.e. leading to the same response as the target) and 50\% were incongruent (leading to the opposite response of the target). Therefore, ignoring the prime would not be a beneficial strategy for the subject, leading to the observed similar priming effects for both prime notations.

The present results support both assumptions made by the GNW model proposed by Dehaene and Naccache (2001). The weak activation evoked by an unconscious stimulus indeed appears to be insufficient to establish a reorganization of the workspace and therefore to be used strategically to enhance task performance (bottom-up effect). Contrarily, a fully consciously perceived stimulus is able to establish a reorganization of the workspace and can therefore be used strategically to enhance task performance (top-down effect). Furthermore, the present study adds to the increasing pile of evidence that unconscious cognitive processes are not merely automatic and independent of attention and strategies. On the contrary, unconscious processing proves to be flexible in nature and sensitive to strategic and contextual influences.

\section{References}

Bodner, G. A., \& Dypvik, A. T. (2005). Masked priming of number judgements depends on prime validity and task. Memory and Cognition, 33, 29-47.

Bodner, G. E., \& Masson, M. E. J. (2001). Prime validity affects masked repetition priming: Evidence for an episodic resource account of priming. Journal of Memory \& Language, 45, 616-647.

Bodner, G. E., \& Masson, M. E. J. (2003). Beyond spreading activation: An influence of relatedness proportion on masked semantic priming. Psychonomic Bulletin \& Review, 10, 645-652.

Dehaene, S., \& Changeux, J. P. (2004). Neural mechanisms for access to consciousness. In M. S. Gazzaniga (Ed.), The cognitive neurosciences (pp. 1145-1157). Cambridge: MIT Press.

Dehaene, S., Changeux, J. P., Naccache, L., Sackur, J., \& Sergent, C. (2006). Conscious, preconscious, and subliminal processing: A testable taxonomy. Trends in Cognitive Sciences, 10, 204-211.

Dehaene, S., \& Naccache, L. (2001). Towards a cognitive neuroscience of consciousness: Basic evidence and a workspace framework. Cognition, 79, 1-37.

Fias, W. (2001). Two routes for the processing of verbal numbers: evidence from the SNARC effect. Psychological Research, 65, 250-259.

Greenwald, A. G., Abrams, R. L., Naccache, L., \& Dehaene, S. (2003). Long-term semantic memory versus contextual memory in unconscious number processing. Journal of Experimental Psychology: Learning, Memory, and Cognition, 29, $235-247$.

Kunde, W., Kiesel, A., \& Hoffmann, J. (2003). Conscious control over the content of unconscious cognition. Cognition, 88, $223-242$.

Naccache, S., Blandin, E., \& Dehaene, S. (2002). Unconscious masked priming depends on temporal attention. Psychological Science, 13, $416-424$.

Perea, M., \& Rosa, E. (2002). Does the proportion of associatively related pairs modulate the associative priming effect at very brief stimulus-onset asynchronies? Acta Psychologica, 110, 103-124.

Reynvoet, B., \& Brysbaert, M. (2004). Cross-notation number priming investigated at different stimulus onset asynchronies in parity and naming tasks. Experimental Psychology, 51, 81-90.

Sumner, P., Tsai, P. C., Yu, K., \& Nachev, P. (2006). Attentional modulation of sensorimotor processes in the absence of perceptual awareness. Proceedings of the National Academy of Sciences of the USA, 103, 10520-10525. 